\section{Kombinationstherapie der Psoriasis vulgaris mit dem 308-nm-Excimer-Laser und Calcipotriol}

\author{
Vorläufiger Erfahrungsbericht
}

\section{B. Kardorff \\ I. Hönig d'Orville \\ M. Wahlen \\ M. Kardorff \\ P. Dorittke}

\author{
Combination Phototherapy of Psoriasis with 308-nm Excimer Laser \\ and Calcipotriol - Preliminary Report
}

\section{Zusammenfassung}

Die Kombinationstherapie mit topischem Calcipotriol und Schmalspektrum UVB $311 \mathrm{~nm}$ gilt als eine der wirksamsten und nebenwirkungsärmsten Therapieformen der mittelschweren Psoriasis vulgaris. Die 308-nm-Excimer-Laser-Monotherapie erreicht nach bisherigen Beobachtungen innerhalb von 8 bis 12 Sitzungen eine etwa $90 \%$ ige Rückbildung der psoriatischen Plaques. An einem Kollektiv von 8 Patienten haben wir die therapeutische Kombination von Calcipotriol mit der 308-nm-Excimer-LaserBestrahlung im Halbseitenversuch gegenüber der 308-nm-Excimer-Laser-Monotherapie bei der Psoriasis vulgaris untersucht und eine deutlich schnellere Remission der Psoriasis auf der Seite der Kombinationstherapie nach bereits 6 bis 9 Sitzungen beobachtet. Ein fotodokumentierter Therapieverlauf wird exemplarisch dargestellt.

\section{Abstract}

Combination phototherapy with calcipotriol and 311-nm narrow band UVB is known as an effective therapy with only little side effects of medium degree psoriasis. 308-nm Excimer Laser monotherapy yields as far as known a $90 \%$ remission of psoriatic plaques within 8 to 12 treatments. In a randomized left-right trial the therapeutic efficacy of calcipotriol in combination with 308-nm Excimer Laser or of Excimer-Laser alone was compared in 8 patients with psoriasis vulgaris. A more rapid remission could be achieved on the combination side within already 6 to 9 treatments. An illustrated case report of one of the eight patients is presented.

\section{Einleitung}

Die Therapie mit dem Excimer-Laser (308 nm) ist eine neuartige Behandlungsmethode der Psoriasis vulgaris [1 -5], die modernste Lasertechnologie und die langjährigen Erfahrungen mit der antipsoriatisch wirksamen UVB-Bestrahlung miteinander kombiniert. Im Gegensatz zur konventionellen UVB-Therapie, bei der etwa 25 - 30 Sitzungen bis zu einer Rückbildung der psoriatischen Plaques erforderlich sind [6-8], wird bei der 308-nm-Excimer-Laser-Therapie nach den bisher vorliegenden Erfahrungen eine Remission der Hautveränderungen nach durchschnittlich 8-12 Behandlungseinheiten erreicht [1 - 3]. Im Rahmen unserer
Untersuchungen zur Effektivität des 308-nm-Excimer-Lasers bei Psoriasis als Monotherapeutikum fiel ein Patient mit deutlich beschleunigtem Ansprechen auf die Therapie aus dem Rahmen. Es stellte sich heraus, dass der Patient aus Eigeninitiative, statt der vereinbarten, die Lasertherapie begleitenden blanden Hautpflege mit einer harnstoffhaltigen Rezeptur, eine parallele topische Behandlung mit calcipotriolhaltiger Salbe durchgeführt hat. Erklärend berichtete er über gute Erfahrungen, die er vor Jahren während eines stationären Aufenthalts mit der Kombinationstherapie Schmalband-UVB $311 \mathrm{~nm}$ und Calcipotriol gemacht hatte [9]. Dies nahmen wir zum Anlass, die Wirksamkeit einer Kombination aus 308-nm-Excimer-Laser-Bestrahlung und 


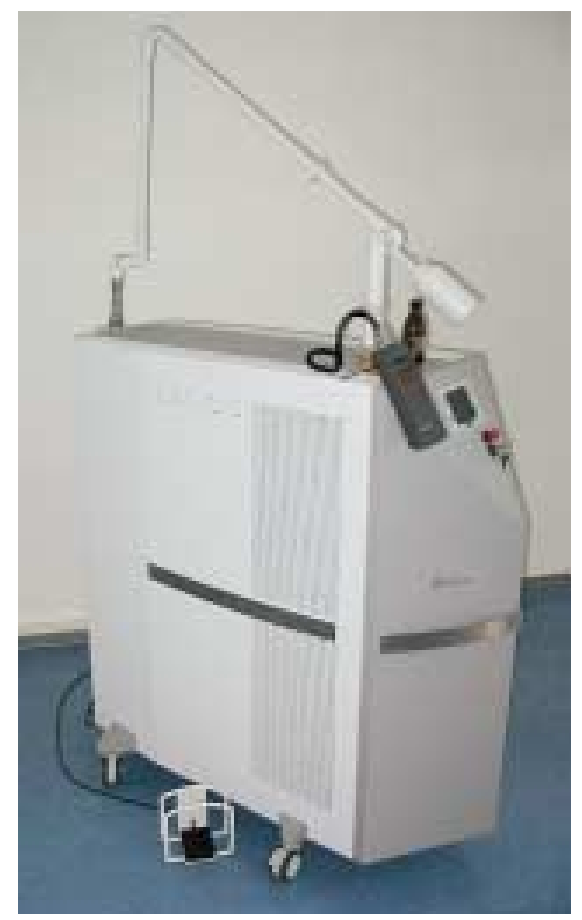

Abb. 1 Excimer-Laser Talos $^{\circledR}: 115 \mathrm{~kg}$, Höhe $125 \mathrm{~cm}$, Tiefe $113 \mathrm{~cm}$, Breite $44 \mathrm{~cm}$; Spiegelgelenkarm, Dosimeter rechts vorne am Laser, Mineralöl-Flasche auf und Fußschalter vor dem Gerät.

topischem Calcipotriol an einem kleinen Kollektiv von 8 Patienten analog der Untersuchungen von Kerscher et al. $[9,10]$ im Halbseitenversuch zu untersuchen. Wir behandelten jedoch beide Körperhälften mit der UVB-308-nm-Laser-Bestrahlung aber nur eine Seite zusätzlich mit topischem Calcipotriol (Daivonex ${ }^{\circledR}$ ). Exemplarisch für die 8 Therapieverläufe berichten wir in nachfolgender fotodokumentierter Falldarstellung über eine 52-jährige Psoriasis-Patientin.

\section{Methode}

Beide Körperhälften wurden mit der UVB-308-nm-Laser-Bestrahlung entsprechend der individuell bestimmten MED und der jeweiligen Plaquedicke bestrahlt mit der Zielsetzung, das Auftreten einer zweitgradigen Dermatitis solaris zu vermeiden, um eine hierdurch bedingte zügigere Abheilung zu umgehen [1 - 3]. Zusätzlich ließen wir $2 \times$ täglich calcipotriolhaltige Salbe (Daivonex ${ }^{\circledR}$-Salbe) halbseitig auftragen. Nur am jeweiligen Morgen vor der Excimer-Laser-Therapie wurde aufgrund der ausgeprägten Lichtfiltereigenschaften des Calcipotriols pausiert. Die andere Körperhälfte wurde mit $5 \%$ igem Harnstoff in Unguentum leniens gepflegt. Zur Beurteilung der Psoriasisherde im Seitenvergleich wurde vor Therapiebeginn und nach jeder zweiten Bestrahlungssitzung sowie bei Erreichen einer fast vollständigen Remission der lokale PSI-Score - ein modifizierter PASI-Score (Psoriasis Area \& Severity Index) ohne Berücksichtigung der befallenen Fläche, nur unter Berücksichtigung von Erythem, Infiltration und Schuppung - jeweils für beide Seiten bestimmt (PSIScore: Psoriasis Severity Index).

\section{Ausschlusskriterien}

Ausschlusskriterien für unsere Untersuchung waren ein Befall der gesamten Körperoberfläche von mehr als $10 \%$ [2,3], da größere Flächen nicht für eine Excimer-Laser-Behandlung geeignet

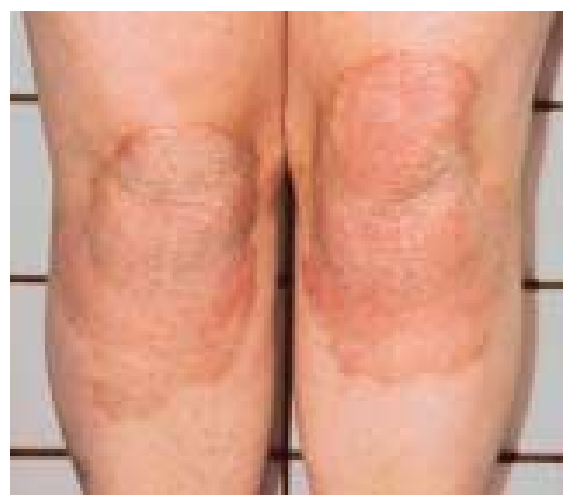

Abb. 2 Erythematosquamöse Plaques beider Knie mit ausgeprägtem Erythem (3 PSI-Score-Punkte), mäßiger Infiltration (2 Punkte) und mäßiger Schuppung (2 Punkte).

sind, eine systemische Psoriasis-Therapie im letzten Jahr, bekannte Hyperkalzämien, Nieren- oder Leberfunktionsstörungen sowie die Einnahme von Kalzium- oder Vitamin-D-Präparaten.

\section{Strahlenquelle}

Talos ${ }^{\circledR}$-XenonChlorid-Excimer-Laser 308 nm (Firma: WaveLight Laser Technologie AG/Erlangen) (Abb.1). Die drei verschiedenen Handstücke haben Durchmesser von 15, 20 und $25 \mathrm{~mm}$ und erfassen Therapieflächen von 1,8, 3,1 oder $4,9 \mathrm{~cm}^{2}$. Durch den kombinierten Einsatz der Handstücke können unterschiedlich große Hautareale auch mit unregelmäßiger Randbegrenzung flächendeckend unter Schonung der nichtbefallenen Haut bestrahlt werden. Die Pulslänge des Talos ${ }^{\circledR}$-Excimer-Lasers beträgt $60 \mathrm{~ns}$ mit einer Repetitionsfrequenz von $200 \mathrm{~Hz}$ und einer Energie pro Puls von 4,6 mJ. Die mittlere Leistungsdichte beträgt $200 \mathrm{~mW} /$ $\mathrm{cm}^{2}$.

\section{Anamnese}

52-jährige Patientin. Psoriasis vom Plaque-Typ an Streckseiten von Armen und Beinen seit dem 24. Lebensjahr. Im letzten halben Jahr erfolgte keine spezifische Therapie der Psoriasis, abgesehen von einem $1 \times$ wöchentlichen häuslichen Solebad mit Totem-Meer-Salz in der eigenen Badewanne und sporadischer Hautpflege mit Melkfett.

\section{Hautbefund}

Erythematosquamöse Plaques vom chronisch stationären Typ finden sich an den Streckseiten von Knien (Abb. 2), Ellenbogen und Unterarmen. Gesamt-PASI-Score vor Therapiebeginn: 9,6. Lokaler PSI (Psoriasis-Severity-Index) der Knie jeweils 7. Hauttyp III nach Fitzpatrick.

\section{Fototherapie}

Zur Bestimmung der MED (Minimale Erythemdosis) wurden 6 benachbarte Stellen unbefallener Haut im Glutäalbereich mit Testdosen von 100,150, 200, 250, 300 und $350 \mathrm{~mJ} / \mathrm{cm}^{2}$ bestrahlt. Die MED, definiert als niedrigste Dosis bei der sich zum Ablesezeitpunkt nach $24 \mathrm{~h}$ ein Erythem zeigt, betrug bei der Patientin $250 \mathrm{~mJ} / \mathrm{cm}^{2}$. Nach Speight 1994 [11] toleriert die psoriatisch veränderte Haut deutlich mehr UV-Strahlung ohne das Auftreten von Blasen, als die gesunde Haut, bei der es ab etwa dem 3fachen der MED zu einer zweitgradigen solaren Dermatitis kommt. Die psoriatischen Läsionen an Knien und Ellenbogen behandelten wir deshalb mit dem 3fachen der gefundenen MED $(3 \times 250 \mathrm{~mJ} /$ $\mathrm{cm}^{2}=750 \mathrm{~mJ} / \mathrm{cm}^{2}$ ) 3-mal wöchentlich bis zur mind. $90 \%$ igen 
Rückbildung im nicht blasenbildenden Dosisbereich. Eine initiale 3-tägige Abschuppungsphase mit 5\%iger Salicylvaseline wurde 3 Tage vor Beginn der Vitamin- $\mathrm{D}_{3}$-Behandlung aufgrund der Inaktivierung des Wirkstoffs ausgesetzt. Linksseitig trug die Patientin $2 \times$ tgl. dünn calcipotriolhaltige Salbe auf (außer am Morgen vor der Bestrahlung), rechtsseitig $2 \times$ tgl. Urea pura $5 \%$ in Unguentum leniens (308-nm-UVB-Gesamtdosis nach 6 Bestrahlungen: $4,5 \mathrm{~J} / \mathrm{cm}^{2}$, bei Therapieende nach 8 Bestrahlungen auf der Kombinationstherapieseite: $6 \mathrm{~J} / \mathrm{cm}^{2}$, bei Therapieende nach 11 Bestrahlungen auf der monotherapeutischen Kontrollseite: $\left.8,25 \mathrm{~J} / \mathrm{cm}^{2}\right)$.

\section{Therapie und Verlauf}

Der PSI-Score auf beiden Seiten vor Beginn der Therapie betrug jeweils 7 (Abb. 2). Bereits nach 6 UVB-Excimer-Laser-Bestrahlungen zeigte sich eine deutliche klinische Differenz (rechtsseitig weiterhin Schuppung des Knies und verstärkte Infiltration) der beiden Seiten. Rechtsseitig (NUR Excimer-Laser) betrug der PSIScore noch 5, linksseitig (Excimer-Laser + Calcipotriol) nur noch 3 (Abb. 3). Der PSI-Score reduzierte sich somit bereits nach den ersten 6 von 8 Bestrahlungen auf der mit der Kombinationstherapie behandelten linken Seite um $57 \%$ und auf der rechten Seite um 29\% innerhalb eines Behandlungszeitraums von lediglich 14 Tagen. Nach 8 Behandlungen war auf der Seite der Kombinationstherapie eine fast vollständige Rückbildung der psoriatischen Plaques erzielt worden, ein gleichwertig zufriedenstellendes Therapieergebnis wurde auf der Gegenseite nach 11 monotherapeutischen Excimer-Laser-Bestrahlungen erreicht.

\section{Ergebnisse}

Die im Rahmen der obigen Falldarstellung erzielten Ergebnisse sind in unserem Kleinkollektiv repräsentativ für alle 8 in dieser Form im Halbseitenvergleich behandelten Patienten (s. Tab.1). Die durchschnittliche Behandlungszahl auf der Seite der Kombinationstherapie (Excimer-Laser + Calcipotriol) betrug 7,5 Sitzungen (6-9 Bestrahlungen), auf der Gegenseite (Excimer-LaserMonotherapie) 10,5 Sitzungen (9-12 Bestrahlungen).

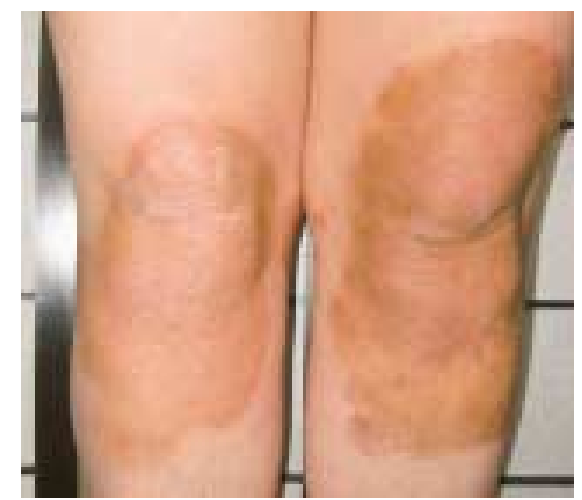

Abb. 3 Knie nach 6 Excimer-Laser-Sitzungen. Rechts: mäßiges Erythem (2 Punkte), mäßige Infiltration (2 Punkte), geringe

Schuppung (1 Punkt). Links nach zusätzlich $2 \times$ täglichem Auftragen von Calcipotriol über 14 Tage: mäßiges Erythem (2 Punkte), geringe Infiltration (1 Punkt), keine Schuppung (0 Punkte), bestrahlungsinduzierte Hyperpigmentierung.

Somit ergibt sich gegenüber herkömmlichen Fototherapieverfahren eine insgesamt deutlich beschleunigte Remission. Die bisherigen Beobachtungen, dass bis zum Erreichen einer $90 \%$ igen Rückbildung der psoriatischen Hautveränderungen 8 - 12 monotherapeutische Excimer-Laser-Sitzungen erforderlich sind [1 -3], konnten wir in unserem Kollektiv mit 9 bis 12 Sitzungen bestätigen. Für das Erreichen dieses sehr guten Therapieergebnisses wurden jedoch durch die beschriebene Kombinationstherapie von topischem Calcipotriol und Excimer-Laser annähernd 30\% weniger Therapiesitzungen benötigt als bei der Monotherapie mit dem 308-nm-Excimer-Laser.

\section{Diskussion}

Eine interessante Weiterentwicklung der UVB-Therapie bei Psoriasis vulgaris stellt der 308-nm-Excimer-Laser [1 -5] dar, welcher seit 3 Jahren in den USA eingesetzt wird. Das monochromatische hochdosierte UVB-Laserlicht wird hierbei exakt auf die

Tab. 1 Zahl der Behandlungen bis zu einer weitgehenden Remission auf der mit der Excimer-Laser-Calcipotriol-Kombinationstherapie behandelten Seite im Vergleich zu der monotherapeutisch mit dem Excimer-Laser behandelten Kontrollseite

\begin{tabular}{|c|c|c|c|c|}
\hline Pat. Nr. & Alter in Jahren & $\begin{array}{l}\text { Geschlecht (M = männlich, } \\
F=\text { weiblich) }\end{array}$ & $\begin{array}{l}\text { Anzahl der Behandlungen } \\
\text { Calcipotriol + Excimer-Laser } 308 \mathrm{~nm}\end{array}$ & $\begin{array}{l}\text { Anzahl der Behandlungen } \\
\text { Excimer-Laser-308-nm-Monotherapie }\end{array}$ \\
\hline 1 & 56 & M & 8 & 10 \\
\hline 2 & 47 & M & 9 & 12 \\
\hline 3 & 53 & $\mathrm{~F}$ & 6 & 10 \\
\hline 4 & 36 & $\mathrm{~F}$ & 7 & 10 \\
\hline 5 & 42 & $M$ & 9 & 11 \\
\hline 6 & 35 & M & 6 & 9 \\
\hline 7 & 48 & $\mathrm{~F}$ & 7 & 11 \\
\hline \multirow[t]{2}{*}{8} & 52 & $\mathrm{~F}$ & 8 & 11 \\
\hline & & & $\varnothing 7,5$ & $\varnothing 10,5$ \\
\hline
\end{tabular}


psoriatischen Läsionen unter Schonung der nicht befallenen Haut appliziert, wodurch der medizinische Therapieerfolg optimiert und das Risiko von Nebenwirkungen gesenkt werden kann. Die Excimer-Laser-Therapie wird in Deutschland derzeit nicht von der gesetzlichen Krankenversicherung erstattet.

Bei dem Laser handelt es sich um einen 308-nm-XeCI(Xenonchlorid)-Excimer-Laser. Das Wort „Excimer“ ist ein Akronym für „excited dimer" und beschreibt ein nur im elektronisch angeregten Zustand existierendes, instabiles zweiatomiges Molekül, welches mit Hilfe von Xenonchloridgas als Katalysator gebildet wird. Es zerfällt schnell in seine beiden Bestandteile - Xenon und Chlorid. Dabei setzt dieses Molekül ein energiereiches Photon frei, wodurch Licht einer ultravioletten Wellenlänge von $308 \mathrm{~nm}$ entsteht. Die $60 \mathrm{~ns}$ kurzen Pulse mit einer Energie von $4,6 \mathrm{~mJ}$ bei einer mittleren Leistungsdichte von $200 \mathrm{~mW} / \mathrm{cm}^{2}$ des von uns verwendeten Systems werden über Handstücke mit Durchmessern von 15, 20 oder $25 \mathrm{~mm}$ appliziert. Durch Kombination der Handstücke wird eine annähernd punktgenaue Bestrahlung der psoriatischen Hautveränderungen ermöglicht [2]. Die bisher veröffentlichten Ergebnisse [1 -5] zu dieser Methode weisen auf eine deutlich beschleunigte Rückbildung der psoriatischen Läsionen sowohl gegenüber konventioneller UV-Therapie als auch gegenüber topischer und systemischer Therapie bei einem Körperoberflächenbefall von bis zu $10 \%$ hin. Nach unseren eigenen, relativ umfangreichen klinischen Erfahrungen mit 2 verschiedenen Excimer-Laser-Systemen spielt die Pulslänge des Einzelimpulses (60 ns gegenüber $30 \mathrm{~ns}$ [1]) für die therapeutische Effektivität keine Rolle. Sowohl die notwendige Zahl der Behandlungen als auch das therapeutische Endergebnis erscheinen klinisch bei beiden Systemen identisch. Durch eine zufällige Beobachtung während der Testphase des Excimer-Lasers als Monotherapeutikum (siehe Einleitung) haben wir ein kleines Kollektiv aus 8 Patienten halbseitig zusätzlich mit Calcipotriol behandelt und eine zusätzliche Beschleunigung des Therapieerfolgs erzielen können.

Calcipotriol ist ein synthetisches Vitamin- $\mathrm{D}_{3}$-Analogon, welches die Zellproliferation hemmt. Psoriasis-Haut zeigt unter Calcipotriol-Therapie eine Reduktion proliferierender Zellen sowie eine Verminderung des mit Hyperproliferation assoziierten Keratins 16. Außerdem fördert es die Zelldifferenzierung [12]. Über positive Therapieeffekte der Kombination von Calcipotriol mit Breitband-UVB und Schmalband UVB $311 \mathrm{~nm}( \pm 2 \mathrm{~nm})$ wurde bereits Anfang der 90er-Jahre des letzten Jahrhunderts berichtet [13]. Die 308-nm-Excimer-Laser-Therapie ist als eine Weiterentwicklung der 311-nm-Schmalspektrum-UVB-Röhren zu sehen, die bereits im Vergleich zur konventionellen UVB/SUP-Therapie ein besseres antipsoriatisches Spektrum bei geringerer erythematogener Potenz aufwiesen. Nach Lehmann [14] gilt die Kombination Calcipotriol/UVB $311 \mathrm{~nm}$ als eine der wirksamsten und nebenwirkungsärmsten Therapieformen der mittelschweren disse- minierten Psoriasis. Die Ursache für das vermutlich noch bessere Ansprechen der Excimer-Laser/Calcipotriol-Kombitherapie gegenüber der 311-nm-Schmalspektrumbehandlung liegt wahrscheinlich in der Möglichkeit einer noch intensiveren Fototherapie. Durch den 308-nm-Excimer-Laser können noch höhere Dosen des antipsoriatisch wirksamen Aktionsspektrums $[15,16]$ in kürzerer Zeit auf die psoriatischen Läsionen unter Schonung der gesunden Haut appliziert werden, und dabei können auch noch die Vorteile der gut verträglichen Fotokombinationstherapie mit Calcipotriol gegenüber der Fotomonotherapie genutzt werden. Unsere hier vorgestellten Erfahrungen mit der Kombinationstherapie der Psoriasis vulgaris mit dem 308-nm-ExcimerLaser und topischem Calcipotriol sind aufgrund des kleinen Kollektivs natürlich noch nicht aussagekräftig. Ausgedehntere klinische Studien sollten diesem ersten Ansatz folgen.

\section{Literatur}

${ }^{1}$ Kardorff B, Hönig d'Orville I, Peters M, Kardorff M, Dorittke P. Monotherapeutikum Excimer Laser $308 \mathrm{~nm}$. Kosmetische Medizin 2001; 22: $290-294$

${ }^{2}$ Kardorff B, Hönig d'Orville I, Kardorff M, Dorittke P. Eximer-Laser $(308 \mathrm{~nm})$ - Therapie einer Patientin mit therapieresistenter Psoriasis vulgaris. Akt Dermatol 2001; 27: 357-360

${ }^{3}$ Asawanonda P, Anderson R, Yuchiao C, Taylor CR. 308-nm Excimer Laser for the treatment of psoriasis. Arch Dermatol 2000; 136: 619-624

${ }^{4}$ Bonis B, Kemeny L, Dobozy A, Bor Z, Szabo G, Ignacz F. 308 nm UVB excimer laser for psoriasis (letter). Lancet 1997; 350: 1522

${ }^{5}$ Kemeny L et al. 308-nm Excimer Laser therapy for psoriasis (letter). Arch Dermatol 2001; 137: 1

${ }^{6}$ Lehmann P. UVB-Therapie. In: Tebbe B, Goerdt S, Orfanos CE. Dermatologie. Heutiger Stand. Stuttgart, New York: Thieme, 1995: 339-341

${ }^{7}$ Krutmann J, Hönigsmann H. Handbuch der Dermatologischen Phototherapie und Photodiagnostik. Berlin: Springer, 1997: 62

${ }^{8}$ Krutmann J et al. Dermatological Phototherapy and Photodiagnostic Methods. Berlin: Springer, 2001: 74

${ }^{9}$ Kerscher M, Plewig G, Lehmann P. Kombinationstherapie der Psoriasis vulgaris mit einem Schmalspektrum UV-B-Strahler (Philips TL 01, $311 \mathrm{~nm}$ ) und Calcipotriol. Akt Dermatol 1994; 20: 151 - 154

${ }^{10}$ Kerscher M, Volkenandt M, Plewig G, Lehmann P. Combination phototherapy of psoriasis with calcipotriol and narrow band UVB. Lancet 1993; 342: 923

11 Speight EL, Farr PM. Erythemal and therapeutic response of psoriasis to PUVA using high-dose UVA. Br J Dermatol 1994; 131: 667-672

${ }^{12}$ Fuhrmeister K. Vitamin-D ${ }_{3}$-Analoga. In: Korting H, Sterry W. Therapeutische Verfahren in der Dermatologie. Berlin: Blackwell, 2001: $139-140$

${ }^{13}$ Kragballe K. Combination of topical calcipotriol and UVB radiation of psoriasis vulgaris. Dermatologica 1990; 181: 211 - 214

14 Lehmann P, Ruzicka T. Neue Entwicklungen in der Phototherapie der Psoriasis. In: Krutmann J, Hönigsmann H. Handbuch der Dermatologischen Phototherapie und Photodiagnostik. Berlin: Springer, 1997: 63

${ }^{15}$ Amlong UJ, Heller J, Schiller F et al. Konzeption einer direkten Phototherapie der Psoriasis unter Berücksichtigung der Überschneidung verschiedener relativer Wirkungsfunktionen. Dermatol Monatsschr 1986; 172: $325-328$

${ }^{16}$ Parrish JA, Jaenicke KF. Action spectrum for phototherapy of psoriasis. J Invest Dermatol 1981; 76: 359-362 